\title{
An Exponential Fourier Series for I-Function of Several Complex Variables
}

\author{
V.G.Gupta ${ }^{1}$, Nawal Kishor Jangid ${ }^{2}$ \\ ${ }^{1}$ Department of Mathematics, University of Rajasthan, Jaipur - 302004, Rajasthan, India
}

${ }^{2}$ Department of Mathematics, Swami Keshvanand Institute of Technology, Management \& Gramothan, Jaipur-302017, Rajasthan, India

\begin{abstract}
In the present paper we established an exponential fourier series for the I-function of several complex variables which is of interest in itself and may find significant usages in the field of applied mathematics. We also record here some special cases of our main findings.
\end{abstract}

Keywords: Multivariable I-function.

AMS Classification: 33C45.

\section{Introduction}

We recall here the definition of I-function of , re variables is given by Prithma Jayarama et al. [5] in the following manner:

$$
\begin{aligned}
& I\left[z_{1}, \ldots, z_{r}\right]= \\
& \underset{p, q: p_{1}, q_{1} ; \ldots ; p_{r}, q_{r}}{0, n: m_{1}, n_{1} ; \ldots ; m_{r}, n_{r}}\left[\begin{array}{c}
z_{1} \\
\vdots \\
z_{r}
\end{array} \mid \begin{array}{c}
\left(a_{j} ; \alpha_{j}^{(1)}, \ldots, \alpha_{j}^{(r)} ; A_{j}\right)_{1, p}:\left(c_{j}^{(1)}, \gamma_{j}^{(1)} ; C_{j}^{(1)}\right)_{1, p_{1}} ; \ldots ;\left(c_{j}^{(r)}, \gamma_{j}^{(r)} ; C_{j}^{(r)}\right)_{1, p_{r}} \\
\left(b_{j} ; \beta_{j}^{(1)}, \ldots, \beta_{j}^{(r)} ; B_{j}\right)_{1, q}:\left(d_{j}^{(1)}, \delta_{j}^{(1)} ; D_{j}^{(1)}\right)_{1, q_{1}} ; \ldots ;\left(d_{j}^{(r)}, \delta_{j}^{(r)} ; D_{j}^{(r)}\right)_{1, q_{r}}
\end{array}\right] \\
& =(2 \pi \omega)^{-r} \int_{L_{1}} \ldots \int_{L_{r}} \phi\left(s_{1}, \ldots, s_{r}\right) \theta_{1}\left(s_{1}\right) \ldots \theta\left(s_{r}\right) z_{1}{ }^{s_{1}} \ldots z_{r}{ }^{s_{r}} d s_{1} \ldots d s_{r} \text {, }
\end{aligned}
$$

here

$$
\phi\left(s_{1}, \ldots, s_{r}\right)=\frac{\prod_{j=1}^{n} \Gamma^{A_{j}}\left[1-a_{j}+\sum_{i=1}^{r} \alpha_{j}^{(i)} s_{i}\right]}{\prod_{j=n+1}^{p} \Gamma^{A_{j}}\left[a_{j}-\sum_{i=1}^{r} \alpha_{j}^{(i)} s_{i}\right] \prod_{j=1}^{q} \Gamma^{B_{j}}\left[1-b_{j}+\sum_{i=1}^{r} \beta_{j}^{(i)} s_{i}\right]}
$$

and

$$
\theta_{i}\left(s_{i}\right)=\frac{\prod_{j=1}^{n_{i}} \Gamma^{C_{j}^{(i)}}\left[1-c_{j}^{(i)}+\gamma_{j}^{(i)} s_{i}\right] \prod_{j=1}^{m_{i}} \Gamma^{D_{j}^{(i)}}\left[d_{j}^{(i)}-\delta_{j}^{(i)} s_{i}\right]}{\prod_{j=n_{i}+1}^{p_{i}} \Gamma^{C_{j}^{(i)}}\left[c_{j}^{(i)}-\gamma_{j}^{(i)} s_{i}\right] \prod_{j=m_{i}+1}^{q_{i}} \Gamma^{D_{j}^{(i)}}\left[1-d_{j}^{(i)}+\delta_{j}^{(i)} s_{i}\right]} ;
$$

where $\mathrm{i}=1, \ldots, \mathrm{r}, z_{i} \neq 0$ and $\omega=\sqrt{-1}$. For more details and convergence conditions, one may refer to [5].

\section{Required Results}

(i) The following formulae are required in the proof :

$$
\int_{0}^{\pi} e^{(2 m+1) i x} \sin (2 n+1) x d x=0, \quad \text { if } m \neq n,
$$

$$
=\pi i / 2, \text { if } m=n,
$$

which has been given in ([1], p. 490, 3,891).

(ii) We shall also need the result contained in the following : Lemma:

$$
\int_{0}^{\pi} \sin (2 k+1) x(\sin x)^{\sigma} I \begin{array}{r}
0, n:\left(m_{1}, n_{1}\right) ; \ldots ;\left(m_{r}, n_{r}\right) \\
p, q:\left(p_{1}, q_{1}\right) ; \ldots ;\left(p_{r}, q_{r}\right)
\end{array}\left[z_{1}(\sin x)^{2 h_{1}}, \ldots, z_{r}(\sin x)^{2 h_{r}}\right] d x
$$




$$
\begin{gathered}
=(-1)^{k} I \begin{array}{r}
0, n+2:\left(m_{1}, n_{1}\right) ; \ldots ;\left(m_{r}, n_{r}\right) \\
p+2, q+2:\left(p_{1}, q_{1}\right) ; \ldots ;\left(p_{r}, q_{r}\right)
\end{array}\left[\begin{array}{c}
z_{1} \\
\vdots \\
z_{r}
\end{array} \mid \begin{array}{r}
\left(1-\frac{\sigma}{2}: h_{1}, \ldots, h_{r} ; 1\right),\left(\frac{1-\sigma}{2}: h_{1}, \ldots, h_{r} ; 1\right), \\
\left(k+\frac{1-\sigma}{2}: h_{1}, \ldots, h_{r} ; 1\right),\left(-k-\frac{1+\sigma}{2}: h_{1}, \ldots, h_{r} ; 1\right), \\
\left(a_{j} ; \alpha_{j}^{(1)}, \ldots, \alpha_{j}^{(r)} ; A_{j}\right)_{1, p}:\left(c_{j}^{(1)}, \gamma_{j}^{(1)} ; C_{j}^{(1)}\right)_{1, p_{1}} ; \ldots ;\left(c_{j}^{(r)}, \gamma_{j}^{(r)} ; C_{j}^{(r)}\right)_{1, p_{r}} \\
\left(b_{j} ; \beta_{j}^{(1)}, \ldots, \beta_{j}^{(r)} ; B_{j}\right)_{1, q}:\left(d_{j}^{(1)}, \delta_{j}^{(1)} ; D_{j}^{(1)}\right)_{1, q_{1}} ; \ldots ;\left(d_{j}^{(r)}, \delta_{j}^{(r)} ; D_{j}^{(r)}\right)_{1, q_{r}}
\end{array}\right]
\end{gathered}
$$

where $\mathrm{k}$ is an integer, $h_{i}>0,1<i<r$, and the convergence conditions for the multivariable I-function are satisfied and

$$
\operatorname{Re}\left(\sigma+2 \sum_{i=1}^{r} h_{i} a_{j}^{(i)} / \delta_{j}^{(i)}\right)>-1 ; j=1, \ldots, u^{(i)}
$$

Proof: To prove the Lemma (2.2), we express the multivariable I-function of several complex variables occurring in the L.H.S. of (2.2) in terms of Mellin-Barnes type contour integral using (1.2), the L.H.S. of (1.3) is as follows:

$\int_{0}^{\pi} \sin (2 k+1) x(\sin x)^{\sigma}\left\{\frac{1}{(2 \pi \omega)^{r}} \int_{L_{1}} \ldots \int_{L_{r}} \phi\left(s_{1}, \ldots, s_{r}\right) \theta_{1}\left(s_{1}\right) \ldots \theta_{r}\left(s_{r}\right)\right.$

$\left.\times z_{1}^{s_{1}}(\sin x)^{2 h_{1} s_{1}}, \ldots, z_{r}^{s_{r}}(\sin x)^{2 h_{r} s_{r}} d s_{1}, \ldots, d s_{r}\right\} d x$

Next, on changing the order of integration the above expression becomes:

$$
\frac{1}{(2 \pi \omega)^{r}} \int_{L_{1}} \ldots \int_{L_{r}} \phi\left(s_{1}, \ldots, s_{r}\right) \theta_{1}\left(s_{1}\right) \ldots \theta_{r}\left(s_{r}\right) z_{1}^{s_{1}} \ldots z_{r}^{s_{r}}
$$

$$
\begin{aligned}
& \times\left\{\int_{0}^{\pi} \sin (2 k+1) x(\sin x)^{\sigma+\sum_{i=1}^{r} 2 h_{i} s_{i}} d x\right\} d s_{1}, \ldots, d s_{r} \\
& \left.\mid \begin{array}{c}
\left(a_{j} ; \alpha_{j}^{(1)}, \ldots, \alpha_{j}^{(r)} ; A_{j}\right)_{1, p}:\left(c_{j}^{(1)}, \gamma_{j}^{(1)} ; C_{j}^{(1)}\right)_{1, p_{1}} ; \ldots ;\left(c_{j}^{(r)}, \gamma_{j}^{(r)} ; C_{j}^{(r)}\right)_{1, p_{r}} \\
\left(b_{j} ; \beta_{j}^{(1)}, \ldots, \beta_{j}^{(r)} ; B_{j}\right)_{1, q}:\left(d_{j}^{(1)}, \delta_{j}^{(1)} ; D_{j}^{(1)}\right)_{1, q_{1}} ; \ldots ;\left(d_{j}^{(r)}, \delta_{j}^{(r)} ; D_{j}^{(r)}\right)_{1, q_{r}}
\end{array}\right] \\
& =\frac{2(-1)^{k}}{\sqrt{\pi i}} \sum_{k=-\infty}^{\infty} \begin{array}{l}
0, n:\left(m_{1}, n_{1}\right) ; \ldots ;\left(m_{r}, n_{r}\right) \\
p,\left(p_{1}, q_{1}\right) ; \ldots ;\left(p_{r}, q_{r}\right)
\end{array}\left|\begin{array}{c}
z_{1} \\
\vdots \\
z_{r}
\end{array}\right| \begin{array}{c}
{\left[-\frac{\sigma}{2}: h_{1}, \ldots, h_{r} ; 1\right],\left[\frac{1-\sigma}{2} ; h_{1}, \ldots, h_{r} ; 1\right]} \\
{\left[k+\frac{\sigma}{2}: h_{1}, \ldots, h_{r} ; 1\right],\left[-k-\frac{1-\sigma}{2} ; h_{1}, \ldots, h_{r} ; 1\right]}
\end{array} \\
& \left.\begin{array}{l}
\left(a_{j} ; \alpha_{j}^{(1)}, \ldots, \alpha_{j}^{(r)} ; A_{j}\right)_{1, p}:\left(c_{j}^{(1)}, \gamma_{j}^{(1)} ; C_{j}^{(1)}\right)_{1, p_{1}} ; \ldots ;\left(c_{j}^{(r)}, \gamma_{j}^{(r)} ; C_{j}^{(r)}\right)_{1, p_{r}} \\
\left(b_{j} ; \beta_{j}^{(1)}, \ldots, \beta_{j}^{(r)} ; B_{j}\right)_{1, q}:\left(d_{j}^{(1)}, \delta_{j}^{(1)} ; D_{j}^{(1)}\right)_{1, q_{1}} ; \ldots ;\left(d_{j}^{(r)}, \delta_{j}^{(r)} ; D_{j}^{(r)}\right)_{1, q_{r}}
\end{array}\right] e^{(2 k+1) i \pi}
\end{aligned}
$$

where $h_{i}>0,1<i<r, 0<x<\pi ; \operatorname{Re}(\sigma)>0$, and the convergence conditions are satisfied. result

$\int_{0}^{\pi}(\sin x)^{\sigma} \sin \lambda x d x= \begin{cases}0, & \text { if } \lambda=2 k, \\ \frac{\sqrt{\pi}[(1-\sigma) / 2]_{k} \Gamma(1+\sigma / 2)}{\Gamma[k+(\sigma+3) / 2]} & \text { if } \lambda=2 k+1\end{cases}$

due to Luke ([3], p.16, eq. (5)) and then reinterpreting the result in terms of Mellin-Barnes type contour integral as the I-function of r-complex variables, the right hand side of (2.2) follows immediately.

\section{Exponential Fourier series}

The exponential series to be established is

$(\sin x)^{\sigma} I \begin{gathered}0, n:\left(m_{1}, n_{1}\right) ; \ldots ;\left(m_{r}, n_{r}\right) \\ p, q:\left(p_{1}, q_{1}\right) ; \ldots ;\left(p_{r}, q_{r}\right)\end{gathered}\left[\begin{array}{c}z_{1}(\sin x)^{2 h_{1}} \\ \vdots \\ z_{r}(\sin x)^{2 h_{r}}\end{array}\right.$$$
\text { Proof: Let }
$$$$
f(x)=(\sin x)^{\sigma} I^{0, n:\left(m_{1}, n_{1}\right) ; \ldots ;\left(m_{r}, n_{r}\right)} \begin{array}{r}
p, q:\left(p_{1}, q_{1}\right) ; \ldots ;\left(p_{r}, q_{r}\right)
\end{array}
$$ 


$$
\begin{aligned}
& {\left[\begin{array}{c}
z_{1}(\sin x)^{2 h_{1}} \\
\vdots \\
z_{r}(\sin x)^{2 h_{r}}
\end{array} \mid \begin{array}{l}
\left(a_{j} ; \alpha_{j}^{(1)}, \ldots, \alpha_{j}^{(r)} ; A_{j}\right)_{1, p}:\left(c_{j}^{(1)}, \ldots, \beta_{j}^{(r)} ; B_{j}\right)_{1, q}:\left(\gamma_{j}^{(1)}, C_{j}^{(1)}\right)_{1, p_{1}} ; \ldots ;\left(\delta_{j}^{(1)} ; D_{j}^{(1)}\right)_{1, q_{1}} ; \ldots ;\left(d_{j}^{(r)}, \delta_{j}^{(r)} ; C_{j}^{(r)}\right)_{1, p_{r}} \\
\left.\quad=\sum_{k=-\infty}^{(r)}\right)_{1, q_{r}} E_{k} e^{(2 k+1) i x},(0<x<\pi)
\end{array}\right]}
\end{aligned}
$$

Equation (3.2) is valid since $f(x)$ is continuous and of bounded variation in the open interval $(0, \pi)$.
Multiplying both sides of (3.2) by $\sin (2 \mathrm{k}+1) \mathrm{x}$ and integrating with respect to $\mathrm{x}$ from 0 to $\pi$ and using (2.1) and (2.2) we obtain

$$
\begin{aligned}
& E_{k}= \\
& \frac{2(-1)^{k}}{\sqrt{\pi i}} \stackrel{0, n+2:\left(m_{1}, n_{1}\right) ; \ldots ;\left(m_{r}, n_{r}\right)}{p+2, q+2:\left(p_{1}, q_{1}\right) ; \ldots ;\left(p_{r}, q_{r}\right)}\left[\begin{array}{c}
z_{1} \\
\vdots \\
z_{r}
\end{array} \mid \begin{array}{c}
{\left[-\frac{\sigma}{2}: h_{1}, \ldots, h_{r} ; 1\right],\left[\frac{1-\sigma}{2} ; h_{1}, \ldots, h_{r} ; 1\right],} \\
{\left[k+\frac{1-\sigma}{2}: h_{1}, \ldots, h_{r} ; 1\right],\left[-k-\frac{1+\sigma}{2} ; h_{1}, \ldots, h_{r} ; 1\right],}
\end{array}\right. \\
& \left.\begin{array}{l}
\left(a_{j} ; \alpha_{j}^{(1)}, \ldots, \alpha_{j}^{(r)} ; A_{j}\right)_{1, p}:\left(c_{j}^{(1)}, \gamma_{j}^{(1)} ; C_{j}^{(1)}\right)_{1, p_{1}} ; \ldots ;\left(c_{j}^{(r)}, \gamma_{j}^{(r)} ; C_{j}^{(r)}\right)_{1, p_{r}} \\
\left(b_{j} ; \beta_{j}^{(1)}, \ldots, \beta_{j}^{(r)} ; B_{j}\right)_{1, q}:\left(d_{j}^{(1)}, \delta_{j}^{(1)} ; D_{j}^{(1)}\right)_{1, q_{1}} ; \ldots ;\left(d_{j}^{(r)}, \delta_{j}^{(r)} ; D_{j}^{(r)}\right)_{1, q_{r}}
\end{array}\right] e^{(2 k+1) i \pi}
\end{aligned}
$$

From (3.2) and (3.3), the formula (3.1) follows immediately.

\section{Special Cases}

1) if we put all the exponents

$A_{j}(j=1, \ldots, p), B_{j}(j=1, \ldots, q)$,

$C_{j}^{(i)}\left(j=1, \ldots, p_{i}, i=1, \ldots, r\right)$, and

$D_{j}^{(i)}\left(j=1, \ldots, q_{i}, i=1, \ldots, r\right)$ in (3.1) are equal to unity,

we obtain the result in terms of $\mathrm{H}$-function of " $\mathrm{r}$ " variables given by Gupta [2].

2) For $r=2$ in the result obtained in case (i), the $\mathrm{H}$-function of several complex variables reduces to $\mathrm{H}$-function of two variables defined by Mittal and Gupta [4] and we get the corresponding Fourier series due to Gupta[2].

\section{References}

[1] Edwards J., A treatise on the integral calculus, Vol. II, Chelsa Pub. Co., New York (1962).

[2] Gupta, V.G.,An exponential Series For Multivariable HFunction, Jnanabha, vol. 14, 1984.

[3] Luke, Y. L.,The special functions and their approximations, Vol. I, Academic Press, New York and London, 1969.

[4] Mittal, P.K., and Gupta, K.C., An integral involving generalized function of two variables. Proc. Indian Acad. Sci. Sect. A(1972). 75, 117-123.

[5] Prathima Jayarama, Vasudevan Nambisan Theke Madam and Shantha Kumari Kurumujji, A Study of I-function of Several Complex Variables, International Journal of Engineering Mathematics Vol.2014(2014). 